PROCEEDINGS OF THE

AMERICAN MATHEMATICAL SOCIETY

Volume 139, Number 6, June 2011, Pages 2093-2104

S 0002-9939(2010)10639-1

Article electronically published on November 10, 2010

\title{
DIRICHLET SETS, ERDÖS-KUNEN-MAULDIN THEOREM, AND ANALYTIC SUBGROUPS OF THE REALS
}

\author{
PETER ELIAŠ
}

(Communicated by Julia Knight)

\begin{abstract}
We prove strengthenings of two well-known theorems related to the Lebesgue measure and additive structure of the real line. The first one is a theorem of Erdős, Kunen, and Mauldin stating that for every perfect set there exists a perfect set of measure zero such that their algebraic sum is the whole real line. The other is Laczkovich's theorem saying that every proper analytic subgroup of the real line is included in an $F_{\sigma}$ set of measure zero. Using the strengthened theorems we generalize the fact that permitted sets for families of trigonometric thin sets are perfectly meager.
\end{abstract}

\section{INTRODUCTION}

This paper is motivated by the study of special sets of harmonic analysis, called 'trigonometric thin sets'. These are sets of real numbers, usually small in the sense of Lebesgue measure and Baire category, which can play the role of a set of exceptions in statements about the convergence of trigonometric series. For example, the well-known Carleson's theorem states that a Fourier series of a $2 \pi$ periodic square-integrable function $f$ converges pointwise to the value of $f$ for all reals $x$, except points from a set of measure zero. Another example is the DenjoyLuzin theorem saying that if a trigonometric series absolutely converges outside a measure zero set, then it must absolutely converge everywhere.

From many types of trigonometric thin sets let us mention here one. A set $X \subseteq \mathbb{R}$ is a Dirichlet set if there exists an increasing sequence of natural numbers $\left\{n_{k}\right\}_{k \in \mathbb{N}}$ such that the sequence of functions $\left\{\sin n_{k} \pi x\right\}_{k \in \mathbb{N}}$ converges uniformly to 0 on $X$. It is not difficult to show that every Dirichlet set is included in a closed (even perfect) Dirichlet set and has Lebesgue measure zero.

In 1981, P. Erdős, K. Kunen, and R. D. Mauldin proved that if $P$ is a nonempty perfect set of reals, then there exists a perfect set $M$ of Lebesgue measure zero such that their algebraic sum $P+M=\{x+y: x \in P \wedge y \in M\}$ is the whole real line 77. The proof was based on a variation of a number-theoretic theorem by G. G. Lorentz. We now present a different proof, based on Kronecker's approximation theorem. We also obtain a somewhat stronger result, finding a perfect set $M$ which is a Dirichlet set.

Received by the editors September 10, 2008 and, in revised form, June 4, 2010.

2010 Mathematics Subject Classification. Primary 28A05, 54H05, 54H11.

Key words and phrases. Dirichlet sets, permitted sets, analytic subgroups of the reals, Kronecker's theorem, Erdős-Kunen-Mauldin theorem.

This work was supported by grant No. 1/0032/09 of Slovak Grant Agency VEGA.

(C)2010 American Mathematical Society Reverts to public domain 28 years from publication 
An immediate consequence of this strengthened Erdős-Kunen-Mauldin theorem is the negative answer to the problem of existence of a perfect permitted set, treated at first by J. Arbault in 1952 [1. By a slight modification of the proof we can even prove that every permitted set is perfectly meager; i.e., it is of first category relative to any perfect set.

In 1998, A. Nowik, M. Scheepers, and T. Weiss introduced the notion of AFC'sets 10. It was shown that this notion is consistently stronger than the notion of perfectly meager sets, and $\mathrm{AFC}^{\prime}$-sets were also called 'perfectly meager in the transitive sense'. We prove that permitted sets also have this stronger property. Further, one can naturally extend the notion of permitted sets relative to a given family of sets. In the next part of this paper we will show that all permitted sets for a family $\mathcal{F}$ of subsets of the reals are perfectly meager in the transitive sense, provided that $\mathcal{F}$ has the following properties: it is closed under taking subsets and generating subgroups of $\mathbb{R}$, it contains all pseudo-Dirichlet sets (this is a slight weakening of the notion of Dirichlet sets; see Definition 2.2), and every set $E \in \mathcal{F}$ can be covered by an $F_{\sigma}$-set $F$ such that $E+F \neq \mathbb{R}$.

The last condition is obviously satisfied by all considered families of trigonometric thin sets and motivated us to further study. In 1998, M. Laczkovich proved that every proper analytic subgroup of $\mathbb{R}$ can be covered by an $F_{\sigma}$-set of Lebesgue measure zero. In the last part of this paper we will show that if $E$ is a proper analytic subgroup of the reals, then there exists an $F_{\sigma}$-set $F \supseteq E$ such that even the set $E+F$ has measure zero, and, moreover, it belongs to the $\sigma$-ideal $\mathcal{E}$ generated by closed measure zero sets. To this end we prove that every analytic subgroup of the real line admits a special Suslin scheme, and use a theorem of S. Solecki about analytic sets and $\sigma$-ideals generated by closed sets [13].

\section{Preliminaries}

The results of this paper are motivated by the study of small sets of reals related to the absolute convergence of trigonometric series. Because of the periodicity of trigonometric functions, it is convenient to work on the unit circle $\mathbb{T}$ instead of the real line $\mathbb{R}$. We prefer the additive notation; i.e., $\mathbb{T}$ denotes the quotient topological group $\mathbb{R} / \mathbb{Z}$, with addition as the group operation. Also, all our results can be stated for both $\mathbb{T}$ and $\mathbb{R}$, and we will not distinguish between these two contexts.

For $x \in \mathbb{T}$, let $\|x\|$ denote the distance between $x$ and 0 , or, when considering the real line, a distance of $x \in \mathbb{R}$ to the nearest integer. Then $\varrho(x, y)=\|x-y\|$ is a metric on $\mathbb{T}$ yielding the quotient topology. Since $\|x\| \leq|\sin \pi x| \leq \pi\|x\|$, we can use $\|x\|$ instead of $\sin \pi x$ when considering the convergence of sequences of trigonometric functions or the absolute convergence of trigonometric series. For example, one can equivalently define Dirichlet sets as follows.

Definition 2.1. A set $X \subseteq \mathbb{T}$ is called a Dirichlet set if there exists an increasing sequence of natural numbers $\left\{n_{k}\right\}_{k \in \mathbb{N}}$ and a sequence of positive reals $\left\{\varepsilon_{k}\right\}_{k \in \mathbb{N}}$ converging to zero such that $\forall x \in X \forall k \in \mathbb{N}\left\|n_{k} x\right\| \leq \varepsilon_{k}$.

It is clear that the above definition remains equivalent if instead of accepting an arbitrary sequence $\left\{\varepsilon_{k}\right\}_{k \in \mathbb{N}}$ converging to zero we require a fixed one, e.g., $\varepsilon_{k}=2^{-k}$. We often use this fact without further notice.

We will also need some other types of trigonometric thin sets. 


\section{Definition 2.2. A set $X \subseteq \mathbb{T}$ is}

(1) a pseudo-Dirichlet set (or $p D$-set) if there exists an increasing sequence of natural numbers $\left\{n_{k}\right\}_{k \in \mathbb{N}}$ such that $\forall x \in X \exists K \forall k \geq K\left\|n_{k} x\right\| \leq 2^{-k}$,

(2) an Arbault set (or $A$-set) if there exists an increasing sequence of natural numbers $\left\{n_{k}\right\}_{k \in \mathbb{N}}$ such that $\left\|n_{k} x\right\| \rightarrow 0$ for every $x \in X$,

(3) an $N$-set if there exists a sequence $\left\{a_{n}\right\}_{n \in \mathbb{N}}$ of nonnegative reals such that $\sum_{n \in \mathbb{N}} a_{n}=\infty$ and for all $x \in X, \sum_{n \in \mathbb{N}} a_{n}\|n x\|<\infty$.

Z. Bukovská 3 proved that pseudo-Dirichlet sets are those sets which can be covered by an increasing sequence of Dirichlet sets.

N-sets are also called 'sets of absolute convergence'. By the original definition, a set of reals is an $\mathrm{N}$-set if there exists a trigonometric series absolutely converging on it but not absolutely converging everywhere. The equivalent characterization used in Definition 2.2 was proved by R. Salem 12 .

Let us denote by $\mathcal{D}, p \mathcal{D}, \mathcal{A}, \mathcal{N}$ the families of all D-sets, pD-sets, A-sets, and $\mathrm{N}$-sets, respectively. It is easy to see that $\mathcal{D} \subseteq p \mathcal{D} \subseteq \mathcal{A}$ and $p \mathcal{D} \subseteq \mathcal{N}$. It is known that all these inclusions are proper and no inclusion between families $\mathcal{A}$ and $\mathcal{N}$ holds true; see [4, [5].

One can easily show that every Dirichlet set is nowhere dense and has Lebesgue measure zero. Moreover, all sets from families $\mathcal{A}$ and $\mathcal{N}$ are meager sets of measure zero (see [4], pp. 468, 471). For N-sets, this is a classical result of A. Denjoy and N. N. Luzin.

Let us recall the following well-known theorem from approximation theory.

Theorem 2.3 (Kronecker's Approximation Theorem). Let $x_{1}, \ldots, x_{n}$ be irrational numbers linearly independent over $\mathbb{Q}$, i.e., if $\sum a_{i} x_{i}=0$ for some $a_{1}, \ldots, a_{n} \in \mathbb{Q}$, then $a_{i}=0$ for every $i$. Let $y_{1}, \ldots, y_{k}$ be arbitrary reals. Then for every $\varepsilon>0$ and every $K$ there exists an integer $k \geq K$ such that for each $i,\left\|k x_{i}-y_{i}\right\|<\varepsilon$.

\section{ERdőS-KunEn-MaUldin THEOREM}

In 1981, P. Erdős, K. Kunen, and R. D. Mauldin proved that if $P$ is a nonempty perfect set of reals, then there exists a perfect set $M$ of Lebesgue measure zero such that their algebraic sum $P+M=\{x+y: x \in P \wedge y \in M\}$ is the whole real line [7. In this section we prove a somewhat stronger result, finding a perfect set $M$ which is a Dirichlet set.

In what follows, by 'perfect set' we will always mean a nonempty one.

Lemma 3.1. Let $P \subseteq \mathbb{R}$ be a perfect set. Then there exists an increasing sequence of natural numbers $\left\{n_{k}\right\}_{k \in \mathbb{N}}$ such that

(a) for every $y \in \mathbb{R}$ there exists a perfect set $Q \subseteq P$ such that for all $q \in Q$ and $k \in \mathbb{N},\left\|n_{k}(q-y)\right\| \leq 2^{-k}$,

(b) for every $y \in \mathbb{R}$, the set $P \cap(D+y)$ is dense in $P$, where $D=\{x \in \mathbb{R}$ : $\left.\exists K \forall k \geq K \quad\left\|n_{k} x\right\| \leq 2^{-k}\right\}$.

Proof. Fix a countable set $\left\{c_{k}: k \in \mathbb{N}\right\} \subseteq P$ dense in $P$. For every $k \in \mathbb{N}$, denote $B_{k}=\left\{\frac{m}{2^{k+1}}: 0 \leq m<2^{k+1}, m \in \mathbb{N}\right\}$. We define an increasing sequence of natural numbers $\left\{n_{k}\right\}_{k \in \mathbb{N}}$, a sequence of positive reals $\left\{\varepsilon_{k}\right\}_{k \in \mathbb{N}}$, and a sequence $\left\{A_{k}\right\}_{k \in \mathbb{N}}$ of finite subsets of $P$ inductively as follows. We start with $A_{0}=\left\{c_{0}\right\}$ and arbitrary $n_{0}$ and $\varepsilon_{0}>0$. Let $k \geq 0$ and let $A_{k}, n_{k}, \varepsilon_{k}$ be defined such that $\varepsilon_{k}>0$ and $A_{k}$ 
is a finite subset of $P$. For every $i \in\{0,1\}$ and every pair $(a, b) \in A_{k} \times B_{k}$, pick $p_{k}^{i}(a, b) \in P$ in such a way that $\left|p_{k}^{i}(a, b)-a\right| \leq \varepsilon_{k} / 2$, and the set

$$
P_{k}=\left\{p_{k}^{i}(a, b): i \in\{0,1\},(a, b) \in A_{k} \times B_{k}\right\}
$$

is linearly independent over $\mathbb{Q}$. This is possible since every neighbourhood of an element of $P$ contains uncountably many elements of $P$. Using Theorem 2.3 find a natural number $n_{k+1}>n_{k}$ such that for all $i \in\{0,1\}$ and $(a, b) \in A_{k} \times B_{k}$, $\left\|n_{k+1} p_{k}^{i}(a, b)-b\right\|<2^{-(k+2)}$. Finally, find a positive real $\varepsilon_{k+1} \leq \varepsilon_{k} / 2$ such that

(1) $\varepsilon_{k+1}<\min \left\{|p-q|: p, q \in P_{k}, p \neq q\right\} / 2$, and

(2) for all $x, i \in\{0,1\}$ and $(a, b) \in A_{k} \times B_{k}$, if $\left|x-p_{k}^{i}(a, b)\right| \leq \varepsilon_{k+1}$, then $\left\|n_{k+1} x-b\right\| \leq 2^{-(k+2)}$.

Put $A_{k+1}=\left\{c_{0}, \ldots, c_{k+1}\right\} \cup P_{k}$.

To prove (a), let $y \in \mathbb{R}$ be arbitrary. For every $k$ there exists $b_{k} \in B_{k}$ such that $\left\|n_{k+1} y-b_{k}\right\| \leq 2^{-(k+2)}$. Let $B(x, \varepsilon)$ denote the closed interval $[x-\varepsilon, x+\varepsilon]$. Put $Q=\bigcap_{k \in \mathbb{N}} \bigcup_{a \in Q_{k}} B\left(a, \varepsilon_{k}\right)$, where $Q_{0}=A_{0}$ and for all $k, Q_{k+1}=\left\{p_{k}^{i}\left(a, b_{k}\right): i \in\right.$ $\left.\{0,1\}, a \in Q_{k}\right\}$.

Clearly every $Q_{k}$ is a finite subset of $P$; hence the set $Q$ is closed. We have $Q \subseteq P$, since $P$ is closed and every element of $Q$ is a limit of a sequence of elements of $P$. For every $k$ we have $\bigcup_{a \in Q_{k}} B\left(a, \varepsilon_{k}\right) \supseteq \bigcup_{a \in Q_{k+1}} B\left(a, \varepsilon_{k+1}\right)$, and thus the set $Q$ is nonempty. Condition (1) and the fact that $Q_{k} \subseteq P_{k}$ ensure that $Q$ has no isolated point. Hence, $Q$ is a perfect subset of $P$.

For all $k$ and $q \in Q$ there exist $a \in Q_{k}$ and $i \in\{0,1\}$ such that $\left|q-p_{k}^{i}\left(a, b_{k}\right)\right| \leq$ $\varepsilon_{k+1}$. Since $Q_{k} \subseteq A_{k}$, from (2) it follows that $\left\|n_{k+1} q-b_{k}\right\| \leq 2^{-(k+2)}$, and hence $\left\|n_{k+1}(q-y)\right\| \leq\left\|n_{k+1} q-b_{k}\right\|+\left\|n_{k+1} y-b_{k}\right\| \leq 2^{-(k+1)}$. Since $\left\|n_{0}(q-y)\right\| \leq 1 / 2$, it follows that $\left\|n_{k}(q-y)\right\| \leq 2^{-k}$ holds true for every $k$.

To prove part (b), let $D=\left\{x \in \mathbb{R}: \exists K \forall k \geq K\left\|n_{k} x\right\| \leq 2^{-k}\right\}$, and let $y$ be a given real. Again, for every $k$, let $b_{k} \in B_{k}$ be such that $\forall k\left\|n_{k+1} y-b_{k}\right\| \leq 2^{-(k+2)}$. We are going to show that $P \cap(D+y)$ is dense in $P$. Let $U$ be an open set intersecting $P$. Then there exist $a \in P$ and $\varepsilon>0$ such that $B(a, \varepsilon) \subseteq U$. Let $K$ be such that $\varepsilon_{K} \leq \varepsilon / 2$ and $c_{K} \in B(a, \varepsilon / 2)$. Now for all $x$, if $\left|x-c_{K}\right| \leq \varepsilon_{K}$, then $x \in U$.

We put $p_{K}=c_{K}$ and by induction choose $p_{k+1} \in A_{k+1}$ for $k \geq K$ such that $\left|p_{k+1}-p_{k}\right| \leq \varepsilon_{k} / 2$ and for all $x$, if $\left|x-p_{k+1}\right| \leq \varepsilon_{k+1}$, then $\left\|n_{k+1} x-b_{k}\right\| \leq 2^{-(k+2)}$. Clearly the sequence $\left\{p_{k}\right\}_{k \in \mathbb{N}}$ has a limit $p \in P$, and for all $k \geq K,\left|p-p_{k}\right| \leq \varepsilon_{k}$. Thus $p \in U$ and if $k \geq K$, then $\left\|n_{k+1}(p-y)\right\| \leq\left\|n_{k+1} p-b_{k}\right\|+\left\|n_{k+1} y-b_{k}\right\| \leq$ $2^{-(k+1)}$. Hence $p-y \in D$, i.e., $p \in D+y$.

It is clear that the above lemma holds true also for $\mathbb{T}$ instead of $\mathbb{R}$. Using part (a) we can now prove the strengthening of the Erdős-Kunen-Mauldin theorem.

Theorem 3.2. For every perfect set $P \subseteq \mathbb{T}$ there exists a perfect Dirichlet set $D$ such that $P+D=\mathbb{T}$.

Proof. For a given perfect set $P$, let $\left\{n_{k}\right\}_{k \in \mathbb{N}}$ be the sequence found in Lemma 3.1 let $E$ be the Dirichlet set $\left\{x \in \mathbb{T}: \forall k\left\|n_{k} x\right\| \leq 2^{-k}\right\}$, and let $D$ be its perfect core, i.e., $D=E \backslash \bigcup\{U: U$ is an open set, $U \cap D$ is countable $\}$. Since by (a), $E$ has a perfect subset, the set $D$ is nonempty and hence a perfect Dirichlet set.

By (a), for every $y \in \mathbb{T}$ there is a perfect set $Q \subseteq P$ such that $Q-y \subseteq E$. We also have $y-Q \subseteq E$ since $E$ is symmetric, and $y-Q \subseteq D$ since $y-Q$ is perfect. Take any $q \in Q$. Then $y-q \in D$, and hence $y=q+(y-q) \in P+D$. 
Part (b) of Lemma 3.1 will be used in the next section.

\section{Permitted SETS}

It should be noted that not one of the considered families of trigonometric thin sets is closed under union (see, e.g., 4, p. 473). In 1952, J. Arbault and independently P. Erdős (see 11, p. 271) proved that a union of an N-set and a countable set is again an $\mathrm{N}$-set. This fact inspired the following definition.

Definition 4.1 (Arbault). A set $A \subseteq \mathbb{T}$ is called permitted if $A \cup X$ is an $\mathrm{N}$-set for any $\mathrm{N}$-set $X$.

Thus, the Arbault-Erdős theorem says that every countable set is permitted. In [1, J. Arbault also claimed that there exists a perfect permitted set. However, in 1961, N. K. Bari found a gap in his proof and stated the existence of a perfect permitted set as an open problem [2] .

Since the family $\mathcal{N}$ is closed under the generating of a subgroup of $\mathbb{T}$, the union $A \cup X$ in the definition of permitted sets may be replaced by the sum $A+X$. Using this fact, J. Lafontaine in 1968 tried to show that no perfect set is permitted [9]. He claimed that for any perfect set $P$ there is an N-set $X$ such that the sum $P+X$ has a positive measure. However, Lafontaine's proof seems to contain a gap too: he proves that $P+X$ is positive with respect to some Borel measure $\mu$ which is a convolution of two other measures, but there is no evidence why $\mu$ should be the standard Lebesgue measure. Without this, $P+X$ may still be an $\mathrm{N}$-set (which must be Lebesgue null), and $P$ may still be a permitted set.

Since every Dirichlet set is also an N-set, Theorem 3.2 shows that Lafontaine's claim was true and hence there exists no perfect permitted set. This fact holds true also in a more general setting.

Definition 4.2. A family of sets $\mathcal{F}$ is called hereditary if it is closed under taking subsets. We say that a set $A$ is $\mathcal{F}$-permitted (see [4, p. 462) if for any $B \in \mathcal{F}$ also $A \cup B \in \mathcal{F}$. If $\mathcal{F}$ is a family of subsets of a group, a set $A$ is $\mathcal{F}$-additive if for any $B \in \mathcal{F}$ also $A+B \in \mathcal{F}$.

The family $\operatorname{Perm}(\mathcal{F})$ of all $\mathcal{F}$-permitted sets is an ideal. If $\mathcal{F}$ is a hereditary family of subsets of a group $G$ and has a base consisting of subgroups of $G$ (i.e., every member of $\mathcal{F}$ is included in a member of $\mathcal{F}$ which is a subgroup of $G$ ), then a set $A \subseteq G$ is $\mathcal{F}$-permitted if and only if it is $\mathcal{F}$-additive.

Corollary 4.3. Let $\mathcal{F}$ be a family of subsets of $\mathbb{T}$ such that $\mathcal{D} \subseteq \mathcal{F}$ and $\mathbb{T} \notin \mathcal{F}$. Then there is no perfect $\mathcal{F}$-additive set. If moreover $\mathcal{F}$ is hereditary and has a base consisting of subgroups of $\mathbb{T}$, then there is no perfect $\mathcal{F}$-permitted set.

Let us note that the families $p \mathcal{D}, \mathcal{A}$, and $\mathcal{N}$ have a basis consisting of subgroups of $\mathbb{T}$.

During 1995-2000, several consistently uncountable examples of permitted sets were constructed by L. Bukovský, M. Repický, T. Bartoszyński, I. Recław, and M. Scheepers (see [4). It seemed that to prove the existence of an uncountable permitted set, one needs to use some additional set-theoretic assumptions. In this situation, L. Bukovský conjectured that every permitted set is perfectly meager; i.e., it is of first category relative to any perfect set. In 2005, we proved this fact for the family of Arbault sets using a combinatorial characterization of the inclusion 
between the sets of the form $\left\{x \in \mathbb{T}:\left\|n_{k} x\right\| \rightarrow 0\right\}$; see [6]. Our version of the Erdős-Kunen-Mauldin theorem now allows a much simpler proof which works also for other families of trigonometric thin sets (Theorem 4.6).

The notion of perfectly meager sets has some natural modifications; see [10], 14.

Definition 4.4 (Zakrzewski). A subset $A$ of a topological space $X$ is called universally meager if for any countable collection $\mathcal{P}$ of perfect subsets of $X$ there exists an $F_{\sigma}$-set $F \supseteq A$ such that $F \cap P$ is meager in $P$ for every $P \in \mathcal{P}$.

Definition 4.5 (Nowik, Weiss). A subset $A$ of a topological group $G$ is perfectly meager in the transitive sense (originally, an $\mathrm{AFC}^{\prime}$-set) if for any perfect set $P \subseteq X$ there exists an $F_{\sigma}$-set $F \supseteq A$ such that for all $y \in G,(F+y) \cap P$ is meager in $P$.

In the topological groups $\mathbb{R}$ and $\mathbb{T}$, every set which is perfectly meager in the transitive sense is also universally meager, and every universally meager set is perfectly meager. The existence of sets contradicting the opposite implications is known to be consistent with ZFC (see [11, [14]).

Theorem 4.6. Let $\mathcal{F}$ be a family of subsets of $\mathbb{T}$ such that $p \mathcal{D} \subseteq \mathcal{F}$ and

(*) for every $E \in \mathcal{F}$ there exists an $F_{\sigma}$-set $F$ such that $E \subseteq F$ and $E+F \neq \mathbb{T}$.

1. Every $\mathcal{F}$-additive set is perfectly meager in the transitive sense.

2. If $\mathcal{F}$ is a hereditary family having a base consisting of subgroups of $\mathbb{T}$, then every $\mathcal{F}$-permitted set is perfectly meager in the transitive sense.

Proof. 1. Let $X$ be $\mathcal{F}$-additive and $P$ be a perfect set. By Lemma 3.1 (b), there exists $D \in p \mathcal{D}$ such that for all $y \in \mathbb{T}, P \cap(D+y)$ is dense in $P$. We have $X-D \in \mathcal{F}$; hence there exists an $F_{\sigma}$-set $F \supseteq X-D$ such that $F-D \neq \mathbb{T}$. It follows that $F \cap(D+x)=\emptyset$ for some $x \in \mathbb{T}$, and hence $P \backslash(F+y) \supseteq P \cap(D+x+y)$ is dense in $P$ for every $y \in \mathbb{T}$. Since $F$ is $F_{\sigma}, P \cap(F+y)$ is meager in $P$ for every $y \in \mathbb{T}$.

2. If $\mathcal{F}$ is hereditary and has a base consisting of subgroups of $\mathbb{T}$, then every $\mathcal{F}$-permitted set is $\mathcal{F}$-additive.

Corollary 4.7. Let $\mathcal{F}$ be any of the families $p \mathcal{D}, \mathcal{A}, \mathcal{N}$. Then every $\mathcal{F}$-permitted set is perfectly meager in the transitive sense.

Proof. It suffices to show that any of the families $p \mathcal{D}, \mathcal{A}, \mathcal{N}$ satisfies the condition $(*)$. This is evident for families $p \mathcal{D}$ and $\mathcal{N}$ since these have a basis consisting of proper $F_{\sigma}$ subgroups of $\mathbb{T}$. In the case of $\mathcal{A}$ we need a different argument.

Let $E$ be an Arbault set, $\left\{n_{k}\right\}_{k \in \mathbb{N}}$ be an increasing sequence of natural numbers such that $\left\|n_{k} x\right\| \rightarrow 0$ for $x \in E$. Put $F=\left\{x \in \mathbb{T}: \exists K \forall k \geq K\left\|n_{k} x\right\| \leq \frac{1}{8}\right\}$. Clearly $F$ is $F_{\sigma}, E \subseteq F$, and $F+F \subseteq \bigcup_{K \in \mathbb{N}}\left\{x \in \mathbb{T}: \forall k \geq K\left\|n_{k} x\right\| \leq \frac{1}{4}\right\}$ is a countable union of nowhere dense sets; hence $E+F \neq \mathbb{T}$.

In the next section we prove that the condition $(*)$ is satisfied for all families having a base consisting of proper analytic subgroups of $\mathbb{T}$.

\section{LACZKOVICH'S THEOREM}

In this section we will use the standard set-theoretic notation: $\omega$ denotes the set of all natural numbers, and $\omega^{\omega}$ and $\omega^{<\omega}$ denote the sets of all infinite and of all finite sequences of natural numbers, respectively. For $t, s \in \omega^{<\omega}, t^{\curlyvee} s$ denotes the concatenation of $t$ and $s, t \subseteq s$ means that $t$ is an initial segment of $s$. For $x \in \omega^{\omega}$ 
and $n \in \omega, x \uparrow n$ is the initial segment of $x$ of length $n$. We denote by $(n)$ the one-element sequence $t \in \omega^{1}$ such that $t(0)=n$.

A subset of a Polish space (i.e., a separable completely metrizable topological space) is called analytic if it is a continuous image of a Borel subset of some other Polish space. It is well known that a set $A$ is analytic if and only if there exists a Suslin scheme for $A$, i.e., an indexed family $\left\{A_{t}: t \in \omega^{<\omega}\right\}$ such that every $A_{t}$ is a closed set, $A_{t} \subseteq A_{s}$ whenever $t \supseteq s$, and $A=\bigcup_{x \in \omega^{\omega}} \bigcap A_{x \uparrow n}$.

We will consider only spaces $\mathbb{R}$ and $\mathbb{T}$ with the standard metric, Lebesgue measure, and with addition as the operation of the topological group.

We say that a Suslin scheme $\left\{A_{t}: t \in \omega^{<\omega}\right\}$ has vanishing diameters if for every $x \in \omega^{\omega}, \lim _{n \rightarrow \infty} \operatorname{diam} A_{x\lceil n}=0$.

Let $\mathcal{E}$ denote the $\sigma$-ideal generated by closed sets of Lebesgue measure zero. Clearly all sets contained in $\mathcal{E}$ are both meager and of measure zero. Moreover, a set belongs to $\mathcal{E}$ if and only if it can be covered by an $F_{\sigma}$-set of measure zero.

M. Laczkovich [8] proved that any proper analytic subgroup of the reals is a subset of an $F_{\sigma}$-set of measure zero. Clearly, this result is also valid for subgroups of $\mathbb{T}$ instead of $\mathbb{R}$. It can thus be formulated as follows.

Theorem 5.1 (Laczkovich). Let $A$ be a proper analytic subgroup of $\mathbb{R}$ or $\mathbb{T}$. Then $A \in \mathcal{E}$.

We will show a somewhat stronger result.

Theorem 5.2. For any proper analytic subgroup $A$ of $\mathbb{R}$ or $\mathbb{T}$ there exists an $F_{\sigma}$-set $F \supseteq A$ such that $A+F \in \mathcal{E}$.

It should be noted here that in the proof of Theorem 5.2 we use the result of Theorem 5.1 as well as the idea of its original proof.

Let us note that by Theorem 3 of [8], there exists a Borel group $G \subseteq \mathbb{R}$ such that $\operatorname{int}(F+F) \neq \emptyset$ for every $F_{\sigma}$-set $F \supseteq G$. Hence, it is impossible to replace condition $A+F \in \mathcal{E}$ in Theorem 5.2 by $F+F \in \mathcal{E}$.

To prove Theorem 5.2, we will use the following result due to S. Solecki [13]. By a portion of a set we mean a nonempty relatively open set.

Theorem 5.3 (Solecki). Let $A$ be an analytic set in a Polish space $X$ and let $\mathcal{I}$ be any $\sigma$-ideal having a base consisting of closed subsets of $X$. Then either $A \in \mathcal{I}$ or there exists a nonempty $G_{\delta}$-set $G \subseteq A$ such that no portion of $G$ belongs to $\mathcal{I}$.

We will also need some lemmas. We work in the unit circle $\mathbb{T}$ or the real line $\mathbb{R}$. The proofs for both spaces are the same.

Lemma 5.4. Let $A$ be an analytic set of measure zero and let $\varepsilon>0$ be arbitrary. Then there exists a Suslin scheme $\left\{B_{t}: t \in \omega^{<\omega}\right\}$ for the set $A$ such that $\sum_{t \in \omega<\omega \backslash\{\emptyset\}} \operatorname{diam} B_{t}<\varepsilon$. Moreover, if $A \in \mathcal{E}$, then we may assume that $B_{t}$ has measure zero for every $t \in \omega^{<\omega} \backslash\{\emptyset\}$.

Proof. Let $\left\{A_{t}: t \in \omega^{<\omega}\right\}$ be a Suslin scheme for $A$. Denote $T=\omega^{<\omega} \backslash\{\emptyset\}$. Since $T$ is countable and $A$ has measure zero, for any $t \in T$ there is a sequence $\left\{I_{j}^{t}\right\}_{j \in \omega}$ of closed sets such that $A \subseteq \bigcup_{j \in \omega} I_{j}^{t}$ and $\sum_{t \in T} \sum_{j \in \omega} \operatorname{diam} I_{j}^{t}<\varepsilon$.

Fix a bijection $\varphi: \omega \times \omega \rightarrow \omega$. For $t \in \omega^{<\omega}$, we define $\psi(t) \in \omega^{<\omega}$ and $B_{t} \subseteq A_{\psi(t)}$ inductively as follows. Put $\psi(\emptyset)=\emptyset$ and $B_{\emptyset}=A_{\emptyset}$. If $\psi(t)$ and $B_{t}$ 
are already defined, for every $k, j \in \omega$ put $\psi\left(t^{\frown} \varphi(k, j)\right)=\psi(t)^{\wedge} k$ and $B_{t^{\frown} \varphi(k, j)}=$ $B_{t} \cap A_{\psi(t) \wedge k} \cap I_{j}^{t^{\wedge} k}$. Let $B=\bigcup_{y \in \omega^{\omega}} \bigcap_{n \in \omega} B_{y\lceil n}$. It is easy to see that $\left\{B_{t}: t \in \omega^{<\omega}\right\}$ is a Suslin scheme, $\sum_{t \in T} \operatorname{diam} B_{t}<\varepsilon$, and $B \subseteq A$.

To see that $A \subseteq B$, assume that $a \in \bigcap_{n \in \omega} A_{x\lceil n}$ for some $x \in \omega^{\omega}$. Let us define $y \in \omega^{\omega}$ by induction as follows. For $n \in \omega$, take $j_{n}$ such that $a \in I_{j_{n}}^{y \nmid n^{-} x(n)}$ and put $y(n)=\varphi\left(x(n), j_{n}\right)$. Then for every $n \in \omega$ we have $\psi(y \uparrow n)=x \uparrow n$ and $B_{y\lceil n+1}=B_{y\lceil n} \cap A_{x\lceil n+1} \cap I_{j_{n}}^{y\left\lceil n^{`} x(n)\right.}$; hence $a \in \bigcap_{n \in \omega} B_{y\lceil n}$.

Now, let us assume that $A \in \mathcal{E}$ and $A \subseteq \bigcup_{i \in \omega} A_{i}$, where the $A_{i}$ are closed sets of measure zero. For every $i$, there exists a Suslin scheme $\left\{A_{t}^{i}: t \in \omega^{<\omega}\right\}$ for the set $A \cap A_{i}$ such that $\sum_{t \in T} \operatorname{diam} A_{t}^{i}<\varepsilon \cdot 2^{-(i+1)}$. Put $B_{\emptyset}^{\prime}=\operatorname{cl}\left(\bigcup A_{\emptyset}^{i}\right)$. For every $t \in T$, define $\tau_{t} \in T$ and $i_{t} \in \omega$ such that $\left|\tau_{t}\right|=|t|, \tau_{t}(n)=t(n)$ for $0<n<|t|$, $t(0)=\varphi\left(i_{t}, \tau_{t}(0)\right)$, and put $B_{t}^{\prime}=A_{i_{t}} \cap A_{\tau_{t}}^{i_{t}}$. Then $\left\{B_{t}^{\prime}: t \in \omega^{<\omega}\right\}$ is a Suslin scheme for the set $A$ and $\sum_{t \in T} \operatorname{diam} B_{t}^{\prime} \leq \sum_{t \in T} \operatorname{diam} A_{\tau_{t}}^{i_{t}}=\sum_{i \in \omega} \sum_{t \in T} \operatorname{diam} A_{t}^{i}<\varepsilon$. Moreover, $B_{t}^{\prime}$ has measure zero for every $t \in T$.

Let us note that if a Suslin scheme $\left\{A_{t}: t \in \omega^{<\omega}\right\}$ satisfies $\sum_{t \in T} \operatorname{diam} A_{t}<\infty$, then it has vanishing diameters.

Lemma 5.5. Let $\left\{A_{t}: t \in \omega^{<\omega}\right\}$ be a Suslin scheme for $A$. Then there exists a Suslin scheme $\left\{B_{t}: t \in \omega^{<\omega}\right\}$ for $A$ such that for all $t \in \omega^{<\omega}, B_{t} \subseteq A_{t}$ and $B_{t} \cap A$ is dense in $B_{t}$.

Proof. For every $t \in \omega^{<\omega}$, let $B_{t}=A_{t} \backslash \bigcup\left\{U: U\right.$ is open, $\left.A_{t} \cap A \cap U=\emptyset\right\}$. Clearly $\left\{B_{t}: t \in \omega^{<\omega}\right\}$ is a Suslin scheme and for all $t \in \omega^{<\omega}, B_{t} \subseteq A_{t}$; hence $\bigcup_{x \in \omega^{\omega}} \bigcap_{n \in \omega} B_{x \mid n} \subseteq A$. If $a \in A$, then $a \in \bigcap_{n \in \omega} A_{x \uparrow n}$ for some $x \in \omega^{\omega}$ and thus $A_{x\lceil n} \cap A \cap U \neq \emptyset$ for each $n$ and every open set $U$ containing $a$. Hence $a \in B_{x \uparrow n}$ for each $n$, and thus $a \in \bigcup_{x \in \omega^{\omega}} \bigcap_{n \in \omega} B_{x\lceil n}$.

For every $t \in \omega^{<\omega}$ we have $B_{t} \supseteq A_{t} \cap A$. Thus, if $B_{t} \cap U \neq \emptyset$ for some open set $U$, then $B_{t} \cap A \cap U \supseteq A_{t} \cap A \cap U \neq \emptyset$. Hence, $B_{t} \cap A$ is dense in $B_{t}$.

Definition 5.6. We say that a set $A$ can be covered by countably many copies of $B$ if there exists a countable set $C$ such that $A \subseteq B+C$. We say that a family $\mathcal{F}$ has the countable covering property if for every $A \in \mathcal{F}$, the set $\{B \in \mathcal{F}$ : $B$ cannot be covered by countably many copies of $A\}$ is finite.

In particular, a Suslin scheme $\left\{A_{t}: t \in \omega^{<\omega}\right\}$ has the countable covering property if for every $t \in \omega^{<\omega}$ there exists a finite set $F_{t} \subseteq \omega^{<\omega}$ such that for every $s \in$ $\omega^{<\omega} \backslash F_{t}$, the set $A_{s}$ can be covered by countably many copies of $A_{t}$.

Let $L(A)$ denote the set of all limit points of the set $A$. We will use the following simple observation.

Lemma 5.7. Let $A$ be a closed subset of a separable metric space $(X, \varrho)$.

1. For every set $B$ such that $A \subseteq L(B)$ there exists a countable set $C \subseteq B$ such that $A=L(C)$.

2. If $A$ is nowhere dense and $B$ is dense, then there exists a countable set $C \subseteq B$ disjoint from $A$ such that $A=L(C)$.

Proof. 1. Let $D$ be a countable dense subset of $A$. Let $\left\{a_{n}\right\}_{n \in \mathbb{N}}$ be a sequence such that for every $d \in D$ there are infinitely many $n$ 's such that $a_{n}=d$. For each $n$ pick some $c_{n} \in B$ so that $\varrho\left(a_{n}, c_{n}\right) \rightarrow 0$. Put $C=\left\{c_{n}: n \in \mathbb{N}\right\}$. If $x \in A$ and $\varepsilon>0$ are arbitrary, then there exists $d \in D$ such that $\varrho(x, d)<\varepsilon / 2$. Further, there exists 
$n$ such that $a_{n}=d$ and $\varrho\left(a_{n}, c_{n}\right)<\varepsilon / 2$; thus $\varrho\left(x, c_{n}\right)<\varepsilon$. Hence $x \in L(C)$. On the other side, if $x \in L(C)$, then there exists an increasing sequence $\left\{n_{k}\right\}_{k \in \mathbb{N}}$ such that $c_{n_{k}} \rightarrow x$. Since $\varrho\left(a_{n_{k}}, c_{n_{k}}\right) \rightarrow 0$, we have $a_{n_{k}} \rightarrow x$; thus $x \in \operatorname{cl} A=A$.

2. Since $B \backslash A$ is dense, we have $A \subseteq L(B \backslash A)$; hence there exists a countable set $C \subseteq B \backslash A$ such that $A=L(C)$.

The key point in the proof of our strengthening of Laczkovich's theorem is the following lemma. Let us note that in the proof of the lemma we use the original version of the theorem.

Lemma 5.8. Let $A$ be a proper analytic subgroup of $\mathbb{R}$ or $\mathbb{T}$. Then there exists a Suslin scheme $\left\{A_{t}: t \in \omega^{<\omega}\right\}$ for $A$ having vanishing diameters, the countable covering property, and such that $A_{t} \cap A$ is dense in $A_{t}$ for all $t \in \omega^{<\omega}$.

Proof. The statement of the lemma is clearly satisfied for countable groups. We will thus assume that $A$ is uncountable, and hence dense in $\mathbb{R}$ or $\mathbb{T}$. By Theorem 5.1 we have $A \in \mathcal{E}$. Denote $T=\omega^{<\omega} \backslash\{\emptyset\}$. By Lemmas 5.4 and 5.5 we can find a Suslin scheme $\left\{B_{t}: t \in \omega^{<\omega}\right\}$ for $A$ such that $\sum_{t \in T} \operatorname{diam} B_{t}<1 / 2, B_{t}$ has measure zero for every $t \in T$, and $B_{t} \cap A$ is dense in $B_{t}$ for every $t \in \omega^{<\omega}$. Moreover, by a suitable pruning we may ensure that each $B_{t}$ is nonempty.

Let us denote by $B(x, r)$ the closed ball with center $x$ and radius $r$. Let $\left\{t_{n}\right\}_{n \in \omega}$ be an enumeration of the set $T$ such that for any $m, n \in \omega$, if $t_{m} \subseteq t_{n}$, then $m \leq n$. Since $A$ is dense, there exist a sequence $\left\{\varepsilon_{n}\right\}_{n \in \omega}$ of positive reals decreasing to 0 and a sequence $\left\{c_{n}\right\}_{n \in \omega}$ of elements of $A$ such that for all $n, B_{t_{n}}+c_{n} \subseteq B\left(0, \varepsilon_{n}\right)$ and $\left(B_{t_{n}}+c_{n}\right) \cap B\left(0, \varepsilon_{n+1}\right)=\emptyset$. Thus the sets $B_{t_{n}}+c_{n}$ are pairwise disjoint, and if $b_{n} \in B_{t_{n}}+c_{n}$ for every $n$, then the sequence $\left\{b_{n}\right\}_{n \in \omega}$ converges to 0 . Hence, for every $n$, the set $C_{n}=\bigcup_{k>n}\left(B_{t_{k}}+c_{k}\right) \cup\{0\}$ is closed. For each $k, B_{t_{k}} \cap A$ is dense in $B_{t_{k}}$; hence the set $\left(B_{t_{k}}+c_{k}\right) \cap A=\left(B_{t_{k}} \cap A\right)+c_{k}$ is dense in $B_{t_{k}}+c_{k}$. Thus, $C_{n} \cap A$ is dense in $C_{n}$, for every $n$.

Fix $m \in \omega$. Let us recall that $(m) \in \omega^{1}$ is a sequence containing only one element, namely $m$. Since $B_{(m)}$ is a closed set of measure zero, it is nowhere dense, and by Lemma 5.7 there exists a countable set $D_{m}=\left\{d_{j}^{m}: j \in \omega\right\} \subseteq A$ disjoint from $B_{(m)}$ and such that $B_{(m)}=L\left(D_{m}\right)$. Since $B_{(m)}$ is closed and no element of $D_{m}$ is in $L\left(D_{m}\right)$, for every $j$ we can choose $\delta_{j}^{m} \in\left\{\varepsilon_{n}: n \in \omega\right\}$ so that $\lim _{j \rightarrow \infty} \delta_{j}^{m}=0$ and the closed balls $B\left(d_{j}^{m}, \delta_{j}^{m}\right)$ are pairwise disjoint and also disjoint from $B_{(m)}$.

By Lemma 5.7, for every $n$ there exists $J_{n} \subseteq \omega$ such that $\min J_{n} \geq n, J_{n^{\prime}} \subseteq J_{n}$ whenever $t_{n^{\prime}} \supseteq t_{n}$, and $L\left(\left\{d_{j}^{m}: j \in J_{n}\right\}\right)=B_{t_{n}}$, where $m=t_{n}(0)$. Put

$$
H_{n}=B_{t_{n}} \cup \bigcup_{j \in J_{n}}\left(B\left(d_{j}^{m}, \delta_{j}^{m}\right) \cap\left(C_{n}+d_{j}^{m}\right)\right),
$$

where $m=t_{n}(0)$. The set $H_{n}$ is closed, since every convergent sequence contained in $H_{n}$ is either almost included in one of the closed sets $B_{t_{n}}$ or $B\left(d_{j}^{m}, \delta_{j}^{m}\right)$ for some $j \in J_{n}$, or its elements meet infinitely many of the sets $B\left(d_{j}^{m}, \delta_{j}^{m}\right), j \in J_{n}$, and hence its limit belongs to $B_{t_{n}}$. The set $B_{t_{n}} \cap A$ is dense in $B_{t_{n}}$, and for all $j$, $\left(C_{n}+d_{j}^{m}\right) \cap A=\left(C_{n} \cap A\right)+d_{j}^{m}$ is dense in $C_{n}+d_{j}^{m}$; hence $H_{n} \cap A$ is dense in $H_{n}$. Also, $H_{n} \in \mathcal{E}$ for every $n$, and $\lim _{n \rightarrow \infty} \operatorname{diam} H_{n}=0$.

We define a family $\left\{A_{t}: t \in \omega^{<\omega}\right\}$ as follows. For all $t \in T$ let $A_{t}=H_{n}$ where $n$ is such that $t=t_{n}$, and let $A_{\emptyset}$ be the closure of $A$, i.e., $\mathbb{R}$ or $\mathbb{T}$. It is easy to see that $\left\{A_{t}: t \in \omega^{<\omega}\right\}$ is a Suslin scheme with vanishing diameters. 
To see that $\bigcup_{x \in \omega^{\omega}} \bigcap_{n \in \omega} A_{x \uparrow n}=\bigcup_{x \in \omega^{\omega}} \bigcap_{n \in \omega} B_{x \uparrow n}=A$, let $x \in \omega^{\omega}$. Since $\lim _{n \rightarrow \infty} \operatorname{diam} A_{x \uparrow n}=0$ and $B_{x \uparrow n} \subseteq A_{x \uparrow n}$ for all $n$, the intersections $\bigcap_{n \in \omega} A_{x \uparrow n}$ and $\bigcap_{n \in \omega} B_{x \uparrow n}$ contain exactly one, hence the same, point.

To show that $\left\{A_{t}: t \in \omega^{<\omega}\right\}$ has the countable covering property, let us take $t \in \omega^{<\omega}$. If $t=\emptyset$, then $A_{t}$ clearly covers every $A_{s}, s \in \omega^{<\omega}$. If $t \in T$, then let $m=t(0), t=t_{n}$, and $j \in J_{n}$. There exists $k^{\prime}$ such that for every $k \geq k^{\prime}$, $B_{t_{k}}+c_{k}+d_{j}^{m}$ is a subset of $B\left(d_{j}^{m}, \delta_{j}^{m}\right)$ and hence also of $A_{t}=H_{n}$. If $l \geq k^{\prime}$, then $A_{t_{l}}$ can be covered by a union of countably many translations of sets $B_{t_{k}}\left(k \geq k^{\prime}\right)$, hence every set $A_{t_{l}}$ such that $l \geq k^{\prime}$ can be covered by countably many copies of $A_{t}$.

Now we can prove the main result of this section.

Proof of Theorem 5.2, Let $A$ be a proper analytic subgroup of $\mathbb{R}$ or $\mathbb{T}$. If $A$ is countable, then the statement is obvious. Let us thus assume that $A$ is uncountable, hence a dense set. By Lemma 5.8, $A$ has a Suslin scheme $\left\{A_{t}: t \in \omega^{<\omega}\right\}$ with vanishing diameters, countable covering property, and such that $A_{t} \cap A$ is dense in $A_{t}$ for all $t \in \omega^{<\omega}$. Denote $T=\omega^{<\omega} \backslash\{\emptyset\}$.

Let $\mathcal{F}$ be the family of all closed sets $F$ such that there exists some $t \in T$ for which the set $F+A_{t}$ has measure zero. The countable covering property implies that if $F \in \mathcal{F}$, then $F+A_{s} \in \mathcal{E}$ for all $s \in \omega^{<\omega}$ except finitely many, and thus $F+A \in \mathcal{E}$. Let $\mathcal{I}$ be the $\sigma$-ideal generated by $\mathcal{F}$. By Theorem 5.3, either $A \in \mathcal{I}$ or there exists a nonempty $G_{\delta}$-set $G \subseteq A$ such that no portion of $G$ is in $\mathcal{I}$.

In the first case we have $A \subseteq F$, where $F=\bigcup_{n \in \omega} F_{n}$ for some $F_{n} \in \mathcal{F}$. Hence $F_{n}+A \in \mathcal{E}$ for every $n, F$ is an $F_{\sigma}$-set, and $F+A \in \mathcal{E}$.

In the rest of the proof we show that the second case is impossible. Assume that $G \subseteq A$ is a nonempty $G_{\delta}$-set such that if $G \cap U \neq \emptyset$ for some open set $U$, then $G \cap U \notin \mathcal{I}$. Let $F$ be the closure of $G$. In what follows, by an interval we will understand a closed connected subset of $\mathbb{R}$ or $\mathbb{T}$ having nonempty interior. For every interval $I$, if $F \cap \operatorname{int} I \neq \emptyset$, then also $G \cap \operatorname{int} I \neq \emptyset$. Therefore, $F \cap I \supseteq G \cap \operatorname{int} I \notin \mathcal{I}$, and thus $(F \cap I)+A_{t}$ has a positive measure, for every $t \in \omega^{<\omega}$. Since there exists an interval $J \subseteq \operatorname{int} I$ such that $F \cap \operatorname{int} J \neq \emptyset$, the same argument shows that also $(F \cap$ int $I)+A_{t}$ has a positive measure, for every $t \in \omega^{<\omega}$.

We will use the same trick as does the proof of Lemma 2 in 8 . Let $I_{0}$ be an arbitrary interval. We shall play the Banach-Mazur game in $I_{0}$ with the second player winning if the intersection of intervals played is a subset of $G+G+A$. We will provide a winning strategy for the second player and this way prove that the set $G+G+A$ is comeager in $I_{0}$. Since $G+G+A \subseteq A$ and $A$ is a meager set, we will obtain a contradiction.

Let $G=\bigcap_{i \in \omega} G_{i}$ where the $G_{i}$ are open. Clearly $G_{i} \cap F$ is dense in $F$, for every $i$. Assume that the first move of the first player is an interval $I_{1} \subseteq I_{0}$. Since $A$ is dense, there exist $x_{1}, y_{1} \in G_{1} \cap F$ and $a_{1}, b_{1} \in A$ such that $x_{1}+a_{1}+y_{1}+b_{1} \in \operatorname{int} I_{1}$. There also exist intervals $J_{1}, K_{1}, L_{1}, M_{1}$ such that $J_{1}, K_{1} \subseteq G_{1}, x_{1} \in \operatorname{int} J_{1}$, $y_{1} \in \operatorname{int} K_{1}, a_{1} \in \operatorname{int} L_{1}, b_{1} \in \operatorname{int} M_{1}$, and $J_{1}+L_{1}+K_{1}+M_{1} \subseteq I_{1}$. There exist $t_{1}, s_{1} \in T$ such that $a_{1} \in A_{t_{1}} \subseteq L_{1}$ and $b_{1} \in A_{s_{1}} \subseteq M_{1}$. Since $F \cap \operatorname{int} J_{1} \neq \emptyset$ and $F \cap \operatorname{int} K_{1} \neq \emptyset$, the sets $\left(F \cap \operatorname{int} J_{1}\right)+A_{t_{1}}$ and $\left(F \cap \operatorname{int} K_{1}\right)+A_{s_{1}}$ have positive measure, and their sum $\left(F \cap \operatorname{int} J_{1}\right)+A_{t_{1}}+\left(F \cap\right.$ int $\left.K_{1}\right)+A_{s_{1}}$ contains an interval $I_{2}$. Clearly $I_{2} \subseteq I_{1}$. Let $I_{2}$ be the response of the second player. 
On the $k^{\text {th }}$ move of the second player, assume that the first player played an interval $I_{2 k-1} \subseteq I_{2 k-2}$, where $I_{2 k-2}$, the response of the second player in the previous move, is an interval contained in $\left(F \cap \operatorname{int} J_{k-1}\right)+A_{t_{k-1}}+\left(F \cap \operatorname{int} K_{k-1}\right)+A_{s_{k-1}}$. Since $G_{k} \cap F$ is dense in $F, A_{t_{k-1}} \cap A$ is dense in $A_{t_{k-1}}$, and $A_{s_{k-1}} \cap A$ is dense in $A_{s_{k-1}}$, there exist $x_{k} \in G_{k} \cap F \cap \operatorname{int} J_{k-1}, y_{k} \in G_{k} \cap F \cap \operatorname{int} K_{k-1}, a_{k} \in$ $A_{t_{k-1}} \cap A, b_{k} \in A_{s_{k-1}} \cap A$ such that $x_{k}+a_{k}+y_{k}+b_{k} \in \operatorname{int} I_{2 k-1}$. There also exist intervals $J_{k}, K_{k}, L_{k}, M_{k}$ with diameters $<1 / k$ such that $J_{k} \subseteq G_{k} \cap J_{k-1}$, $K_{k} \subseteq G_{k} \cap K_{k-1}, x_{k} \in \operatorname{int} J_{k}, y_{k} \in \operatorname{int} K_{k}, a_{k} \in \operatorname{int} L_{k}, b_{k} \in \operatorname{int} M_{k}$, and $J_{k}+L_{k}+K_{k}+M_{k} \subseteq I_{2 k-1}$. There exist $t_{k} \supseteq t_{k-1}, s_{k} \supseteq s_{k-1}$ such that $a_{k} \in A_{t_{k}} \subseteq L_{k}$ and $b_{k} \in A_{s_{k}} \subseteq M_{k}$. Since $F \cap \operatorname{int} J_{k} \neq \emptyset$ and $F \cap \operatorname{int} K_{k-1} \neq \emptyset$, the sets $\left(F \cap \operatorname{int} J_{k}\right)+A_{t_{k}}$ and $\left(F \cap \operatorname{int} K_{k-1}\right)+A_{s_{k}}$ have positive measure, and hence their sum $\left(F \cap\right.$ int $\left.J_{k}\right)+A_{t_{k}}+\left(F \cap\right.$ int $\left.K_{k-1}\right)+A_{s_{k}}$ contains an interval $I_{2_{k}}$. Clearly $I_{2 k} \subseteq I_{2 k-1}$. Let $I_{2 k}$ be the response of the second player.

Now, let $\left\{I_{k}\right\}_{k \in \omega}$ be a sequence of intervals played in a game respecting the described strategy, and let $x_{k}, y_{k}, a_{k}, b_{k}, J_{k}, K_{k}, L_{k}, M_{k}, t_{k}, s_{k}$ be as defined in the $k^{\text {th }}$ move of the second player. There exist unique $x \in \bigcap_{k \in \omega} J_{k}, y \in \bigcap_{k \in \omega} K_{k}$, $a \in \bigcap_{k \in \omega} L_{k}, b \in \bigcap_{k \in \omega} M_{k}$. Moreover, $x_{k} \rightarrow x, y_{k} \rightarrow y, a_{k} \rightarrow a, b_{k} \rightarrow b$. Since for all $k, J_{k} \subseteq G_{k}$ and $K_{k} \subseteq G_{k}$, we have $x, y \in G$. Also, $a \in \bigcap_{k \in \omega} A_{t_{k}}$ and $b \in \bigcap_{k \in \omega} A_{s_{k}}$; hence $a, b \in A$. Since $\operatorname{diam} I_{2 k}<\operatorname{diam} J_{k}+\operatorname{diam} L_{k}+\operatorname{diam} K_{k}+$ $\operatorname{diam} M_{k} \rightarrow 0$, there exists a unique $z \in \bigcap_{k \in \omega} I_{k}$, and $x_{k}+a_{k}+y_{k}+b_{k} \rightarrow z$. Hence, $z=x+a+y+b \in G+G+A$.

The following statement follows directly from Theorems 4.6, 5.2 and the fact that $\mathbb{T} \notin \mathcal{E}$.

Corollary 5.9. Let $\mathcal{F}$ be a family having a base consisting of proper analytic subgroups of $\mathbb{T}$ and let $p \mathcal{D} \subseteq \mathcal{F}$.

1. Then every $\mathcal{F}$-additive set is perfectly meager in the transitive sense.

2. If $\mathcal{F}$ is hereditary then every $\mathcal{F}$-permitted set is perfectly meager in the transitive sense.

It can be shown that every $\mathrm{pD}$-set is included in a group generated by a $\mathrm{D}$-set. Hence, the assumption $p \mathcal{D} \subseteq \mathcal{F}$ in the above theorem can be relaxed to $\mathcal{D} \subseteq \mathcal{F}$. However, the proof of this fact is outside of the scope of this paper.

Let us conclude with an open problem related to Theorem 5.2. If $A$ is a proper analytic subgroup of $\mathbb{T}$ or $\mathbb{R}$, then by Theorem 5.2 there exists an $F_{\sigma}$-set $F \supseteq A$ such that $A+F \in \mathcal{E}$. Hence there exists $x \notin A+F$, and thus $F \cap(A+x)=\emptyset$. In other words, the $F_{\sigma}$-set $F$ separates the group $A$ from its coset $A+x$. Thus, every proper analytic subgroup of $\mathbb{T}$ or $\mathbb{R}$ can be separated by an $F_{\sigma}$-set from one of its cosets. A question is whether this can be true for all cosets.

Problem 5.10. Let $A$ be a proper analytic subgroup of $\mathbb{T}$ or $\mathbb{R}$ and let $x \notin A$. Does there exist an $F_{\sigma}$-set separating the group $A$ from its coset $A+x$ ?

\section{ACKNowledgement}

The author would like to thank the referee for a very careful review of this work. 


\section{REFERENCES}

[1] Arbault J., Sur l'ensemble de convergence absolue d'une série trigonométrique, Bull. Soc. Math. France 80 (1952), 253-317. MR0055476 (14:1080d)

[2] Bari N.K., Trigonometric series, Gosudarstv. Izdat. Fiz.-Mat. Lit., Moscow, 1961, 936 pp. MR0126115 (23:A3411)

[3] Bukovská Z., Thin sets in trigonometrical series and quasinormal convergence, Math. Slovaca 40 (1990), 53-62. MR1094972 (92b:43010)

[4] Bukovský L., Kholshchevnikova N.N., Repický M., Thin sets of harmonic analysis and infinite combinatorics, Real Anal. Exchange 20 (1994/95), 454-509. MR.1348075 (97b:43004)

[5] Eliaš P., Covering for category and trigonometric thin sets, Proc. Amer. Math. Soc. 131 (2003), 3241-3249. MR1992865 (2004d:03102)

[6] Eliaš P., Arbault permitted sets are perfectly meager, Tatra Mt. Math. Publ. 30 (2005), 135148. MR2190254 (2007e:43005)

[7] Erdős P., Kunen K., Mauldin R.D., Some additive properties of sets of real numbers, Fund. Math. 113 (1981), 187-199. MR641304 (85f:04003)

[8] Laczkovich M., Analytic subgroups of the reals, Proc. Amer. Math. Soc. 126 (1998), 17831790. MR1443837(98g:04001)

[9] Lafontaine J., Réunions d'ensembles de convergence absolue, Mém. Soc. Math. France 19 (1969), 21-25.

[10] Nowik A., Scheepers M., Weiss T., The algebraic sum of sets of real numbers with strong measure zero sets, J. Symbolic Logic 63 (1998), 301-324. MR.1610427 (99c:54049)

[11] Nowik A., Weiss T., Not every Q-set is perfectly meager in the transitive sense, Proc. Amer. Math. Soc. 128 (2000), 3017-3024. MR1664434 (2000m:03116)

[12] Salem R., The absolute convergence of trigonometrical series, Duke Math. J. 8 (1941), 317334. MR0004325 (2:360f)

[13] Solecki S., Covering analytic sets by families of closed sets, J. Symbolic Logic 59 (1994), 1022-1031. MR1295987 (95g:54033)

[14] Zakrzewski P., Universally meager sets, Proc. Amer. Math. Soc. 129 (2001), 1793-1798. MR.1814112 (2001m:03097)

Mathematical Institute, Slovak Academy of Sciences, Grešákova 6, Košice, Slovakia E-mail address: elias@upjs.sk 